\title{
Priority Plan for Invasive Cardiac Electrophysiology Procedures During the Coronavirus Disease 2019 (COVID-19) Pandemic
}

\author{
Norman C. Wang et al. ${ }^{1}$ \\ ${ }^{1}$ Affiliation not available
}

April 28, 2020

Published in Journal of Cardiovascular Electrophysiology. DOI forthcoming.

\section{Author list and affiliation}

Norman C. Wang, MD, MS; Sandeep K. Jain, MD; N.A. Mark Estes III, MD; William W. Barrington, MD; Raveen Bazaz, MD; Aditya Bhonsale, MD, MHS; Krishna Kancharla, MBBS; Alaa A. Shalaby, MD, MSc; Andrew H. Voigt, MD; Samir Saba, MD

Heart and Vascular Institute, University of Pittsburgh Medical Center, Pittsburgh, Pennsylvania

Author disclosures: Dr. Wang serves as a consultant for Abbott. Dr. Jain serves as a consultant for Medtronic, receives research support from Medtronic, and is a research investigator for Abbott, Boston Scientific, and Medtronic. Dr. Estes serves as a consultant for Abbott, Boston Scientific, and Medtronic. Dr. Saba receives research support from Abbott and Boston Scientific. The remaining authors have nothing to disclose. University of Pittsburgh Medical Center receives institutional cardiac electrophysiology fellowship support from Abbott, Boston Scientific, and Medtronic.

Funding: This manuscript did not receive any specific grant(s) from funding agencies in the public, commercial, or not-for-profit sectors.

Proposed Tweet: Priority plan for invasive cardiac EP procedures during the COVID-19 pandemic. \#EPeeps \#COVID19

\section{ABBREVIATIONS}

ACC American College of Cardiology

AHA American Heart Association

CDC Centers for Disease Control and Prevention

CMS Centers for Medicare \& Medicaid Services

HRS Heart Rhythm Society

ICD implantable cardioverter-defibrillator

PPE personal protective equipment

SARS-CoV-2 severe acute respiratory syndrome coronavirus 2

INTRODUCTION 
In late December 2019, the Chinese Center for Disease Control and Prevention initiated an investigation of patients with a respiratory illness of unknown etiology in Wuhan. ${ }^{1}$ The causative pathogen was a new coronavirus, severe acute respiratory syndrome coronavirus 2 (SARS-CoV-2), and the disease was designated coronavirus disease 2019, or COVID-19. Two salient features were an overall case-fatality rate of $2.3 \%$ and fast geographical dissemination. ${ }^{2}$ It was stated, "COVID-19 rapidly spread from a single city to the entire country in just 30 days." The initial cluster was theorized to be due to zoonotic transmission from a seafood and wet animal wholesale market. Subsequent person-to-person transmission is believed to occur in a similar fashion to SARS-CoV-1, which caused the SARS outbreak in 2003. ${ }^{3,4}$ This involves contact with infected respiratory droplets, aerosols, and fomites.

In the United States of America, patient zero was a 35-year-old man who presented to an urgent care clinic in the State of Washington on January 19, 2020 after visiting Wuhan. ${ }^{5}$ Given the trajectory of cases and the impending pandemic, the Centers for Disease Control and Prevention (CDC) suggested on February 29 that inpatient facilities should "Reschedule elective surgeries as necessary." ${ }^{6}$ On March 13, the American College of Surgeons recommended that every "hospital, health system, and surgeon should thoughtfully review all scheduled elective procedures with a plan to minimize, postpone, or cancel electively scheduled operations, endoscopies, or other invasive procedures until we have passed the predicted inflection point in the exposure graph and can be confident that our health care infrastructure can support a potentially rapid and overwhelming uptick in critical patient care needs." ${ }^{7}$ Vice Admiral Jerome M. Adams, MD, the Surgeon General, concurred. On March 18, the Centers for Medicare \& Medicaid Services (CMS) issued "guidance to limit non-essential adult elective surgery and medical and surgical procedures" and referenced the Elective Surgery Acuity Scale. ${ }^{8}$

On March 20, a Heart Rhythm Society (HRS) COVID-19 Task Force message agreed with "CDC recommendations to postpone elective EP procedures." ${ }^{9}$ It continued, "Elective procedures may include, but are not limited to, ablation in clinically stable patients, device upgrades, most primary prevention ICD implants, left atrial appendage closure device implants, and implantable loop recorders." On March 31, a guidance paper was published by the HRS COVID-19 Task Force, the American College of Cardiology (ACC) Electrophysiology Council, and the American Heart Association (AHA) Electrocardiography and Arrhythmias Committee. ${ }^{10}$ It divided invasive cardiac electrophysiology procedures into three tiers: 1) urgent/non-elective, 2) semi-urgent, and 3) non-urgent/elective. The purpose of this manuscript is to provide a priority plan for invasive cardiac electrophysiology procedures during the COVID-19 pandemic that is consistent with, yet simplified in comparison to, prior recommendations. ${ }^{6-10}$

\section{SAFETY AND RESOURCE UTILIZATION}

The primary population-level intervention to combat COVID-19 is social distancing. Slowing dissemination of SARS-CoV-2 has been challenging as an estimated $80 \%$ of infected individuals are asymptomatic or mildly symptomatic yet may still shed the virus. ${ }^{2}$ SARS-CoV-2 can survive on surfaces, particularly plastic and stainless steel, for up to 72 hours. ${ }^{3}$ Lack of widespread availability of accurate testing has complicated numerical estimates of cases. Minimizing exposure time to high-risk environments is essential. Family members and healthcare workers in close contact with infected individuals are high-risk groups. ${ }^{2}$ The 3 at-risk groups when considering invasive cardiac electrophysiology procedures are patients, the general population, and hospital personnel.

For patients without COVID-19, the potential benefit of a procedure must be balanced with additional interactions with hospital personnel and time in the hospital. Older age and co-existing medical conditions are associated with increased mortality risk and should be taken into consideration. ${ }^{2}$ Undiagnosed asymptomatic or minimally symptomatic hospital personnel with COVID-19 may still be working. While a goal of a procedure may be to shorten hospitalization time or to decrease the risk of rehospitalization, complications may markedly increase hospital stay and, consequently, infection risk. Acquisition of SARS-CoV-2 may also increase exposure to the general population after discharge.

Personnel within the cardiac electrophysiology laboratory should consult with their in-hospital infection 
prevention and control section for institution-specific considerations. The minimum number of personnel should be involved with patients with known or suspected COVID-19, and trainee participation is discouraged. All workers should be trained and fitted for personal protective equipment (PPE). N95 masks or powered air-purifying respirators are recommended for COVID-19 cases. ${ }^{9,10}$ PPE utilization must account for limited supplies that may be needed later for worst-case scenarios. Increasing availability of SARS-CoV-2 testing should allow more accurate stratification of PPE needs in the procedure planning phase. A major potential consequence of exposure to the cardiac electrophysiology team is that quarantine would make them temporarily unavailable.

Hospitals in geographical regions with a high prevalence of COVID-19, or "hot zones", have reported shortages in PPE, intensive care unit beds, and mechanical ventilators. In these locations, the threshold to consider invasive cardiac electrophysiology procedures may increase even higher. Certain procedures may strain personnel and equipment from various sections. For example, catheter ablation for atrial fibrillation may involve anesthesia and cardiac imaging. Procedurally related adverse events that require intensive care unit monitoring and mechanical ventilation may jeopardize resources needed to treat COVID-19 patients, particularly if a local surge develops. As organizations have called for stoppage to elective procedures ${ }^{6-10}$, this may result in liability.

\section{PRIORITY PLAN}

The overarching principle is that all invasive cardiac electrophysiology procedures that can be reasonably postponed without compromising patient safety should be, until further guidance is available. It is unclear how long postponement of elective procedures will continue to be advised. Reasonable non-invasive options that allow for expedited discharge are preferred.

Challenges for cardiac electrophysiology, like many medical and surgical specialties, are present when differentiating elective versus non-elective procedures. ${ }^{10}$ However, guidelines and consensus statements have been issued by ACC, AHA, and HRS. These have standardized systems that categorize therapies by "Classification of Recommendation." 11 The following priority plan, presented in the Figure , builds upon these documents.

The first consideration, "Guideline or Consensus Statement Classification of Recommendation", allows for the assessment of a multitude of procedures already systematically studied by several guideline and consensus statement committees. It avoids the need for an exhaustive list of procedures and clinical scenarios. The second consideration, "Anticipated Short-term Morbidity or Mortality Benefit", allows for tailoring to the unique clinical characteristics and presentations of each patient. A 30- to 90-day definition of "shortterm" may be adjusted based on individual scenarios and projected time frames to reschedule procedures. Procedures and scenarios with class IIb recommendations, by definition, have weak support, so there are no circumstances to expect high short-term benefit. This priority plan allows for adoption by other countries and regions with their own guidelines and consensus statements that use a similar "Classification of Recommendation" system. ${ }^{12}$ It may also be used or adapted for future pandemics.

Pacemaker and implantable cardioverter-defibrillator (ICD) generator changes are not addressed well in guidelines. Devices within the elective replacement interval should be considered emergent/urgent or equivocal, depending on the indication and the estimated remaining battery life. Same-day discharges for new implants may be encouraged to lower inpatient time and resource utilization.

Hospitalization status may have some bearing on determining potential benefit, particularly if an arrhythmia incited the index hospitalization and may recur in the near future. Hospitalization per se is not a justification for recommending expedited therapy as some may be safely discharged at low risk.

Legal issues should be considered since CMS recommendations were issued. ${ }^{8}$ Hospitals were investigated and fined by the United States Department of Justice nearly one decade ago, in part due to violating CMS policies for the primary prevention ICD. Gross violations to the "no elective procedures" recommendation during the COVID-19 pandemic could conceivably trigger investigations. Proactively documenting the rationale for 
non-elective procedures is worth considering.

\section{Priority I. Emergent/Urgent}

Procedures and scenarios in this category have class I or IIa recommendations with patients at high shortterm risk for mortality or major morbidity. Examples include catheter ablation for medically refractory monomorphic ventricular tachycardia in nonischemic cardiomyopathy; catheter ablation for Wolff-ParkinsonWhite Syndrome associated with rapid atrial fibrillation and syncope; permanent pacemaker for symptomatic complete heart block; and lead extraction for an infected cardiovascular implantable electronic device in the setting of persistent bacteremia. A secondary prevention ICD has a class I recommendation and is preferred prior to discharge. However, a temporary wearable cardioverter-defibrillator may be a reasonable option to explore given unique circumstances that may arise from the COVID-19 pandemic.

\section{Priority II. Equivocal}

Equivocal procedures and scenarios should ideally be postponed. There may be rare circumstances where a compelling reason exists if there is an intermediate chance for short-term benefit due to a substantial nonmortality risk, such as rehospitalization. Preventing rehospitalization is a reasonable goal to decrease risk of infection via social distancing. These should likely only be considered where the prevalence of COVID-19 is low and hospitals do not have shortages of intensive care unit beds and mechanical ventilators. Structured follow-up for patients with procedures put on hold is essential.

Catheter ablation for antiarrhythmic-refractory persistent rapid atrial fibrillation with heart failure and recent recurrent hospitalizations, particularly possible tachycardia-induced cardiomyopathy, is a situation that may be considered non-elective. Conversely, catheter ablation for amiodarone-refractory ischemic ventricular tachycardia with mild-to-moderate symptoms that terminated with antitachycardia pacing has a class I recommendation ${ }^{11}$, but may be elective.

\section{Priority III. Elective}

The potential benefits for procedures and scenarios in this category are long-term. Some may have medical options that are reasonable alternatives. The risk for short-term morbidity or mortality is low. Such situations include left atrial appendage occlusion (although recent stroke or major bleed may elevate the priority to equivocal); catheter ablation for symptomatic idiopathic premature ventricular complexes; catheter ablation for rate-controlled but symptomatic atrial fibrillation or flutter; most primary prevention ICDs; and permanent pacemaker for symptomatic chronotropic incompetence without syncope or near-syncope.

\section{CONCLUSIONS}

The priority plan for invasive cardiac electrophysiology procedures presented here is a flexible yet organized method to facilitate triage during the COVID-19 pandemic. Procedure priority should be decided proactively and with social distancing in mind. Uncertainty or disagreement should be adjudicated by a formal institution-specific process. This simplified approach could also aid in preparations for emergencies that may strain medical resources in the future and in other countries. It should assist in navigating current recommendations by $\mathrm{CDC}$, CMS, and $\mathrm{HRS} / \mathrm{ACC} / \mathrm{AHA}$ to temporarily delay elective procedures towards the goal of flattening the COVID-19 pandemic curve.

\section{REFERENCES}

1. Zhu N, Zhang D, Wang W, et al. A novel coronavirus from patients with pneumonia in China, 2019. $N$ Engl J Med . 2020;382:727-733.

2. Wu Z, McGoogan JM. Characteristics of and important lessons from the Coronavirus Disease 2019 (COVID-19) outbreak in China: Summary of a report of 72314 cases from the Chinese Center for Disease Control and Prevention. JAMA . Published online February 24, 2020. doi:10.1001/jama.2020.2648

3. van Doremalen N, Bushmaker T, Morris DH, et al. Aerosol and surface stability of SARS-CoV-2 as compared with SARS-CoV-1. N Engl J Med . Published online March 17, 2020. doi:10.1056/NEJMc2004973 
4. Cheng VCC, Chan JFW, To KKW, Yuen KY. Clinical management and infection control of SARS: Lessons learned. Antiviral Res . 2013;100:407-419.

5. Holshue ML, DeBolt C, Lindquist S, et al. First case of 2019 novel coronavirus in the United States. $N$ Engl J Med . 2020;382:929-936.

6. Centers for Disease Control and Prevention. Coronavirus disease 2019 (COVID-19): Interim guidance for healthcare facilities. February 29, 2020. Available https://www.cdc.gov/coronavirus/2019ncov/healthcare-facilities/guidance-hcf.html Accessed: March 21, 2020.

7. American College of Surgeons. COVID-19: Recommendations for management of elective surgical procedures. March 13, 2020. Available at: https://www.facs.org/about-acs/covid-19/information-forsurgeons/elective-surgery Accessed: March 21, 2020.

8. Centers for Medicare \& Medicaid Services. CMS releases recommendations on adult elective surgeries, non-essential medical, surgical, and dental procedures during COVID-19 response. March 18, 2020. Available at: https://www.cms.gov/newsroom/press-releases/cms-releases-recommendationsadult-elective-surgeries-non-essential-medical-surgical-and-dental Accessed: March 21, 2020.

9. Heart Rhythm Society. HRS COVID-19 Task Force Message. March 20, 2020. Available at: https://www.hrsonline.org/ Challenges-Solutions/Message01 Accessed March 21, 2020.

10. Lakkireddy DR, Chung MK, Gopinathannair R, et al. Guidance for cardiac electrophysiology during the coronavirus (COVID-19) pandemic from the Heart Rhythm Society COVID-19 Task Force; Electrophysiology Section of the American College of Cardiology; and the Electrocardiography and Arrhythmias Committee of the Council on Clinical Cardiology, American Heart Association. Circulation . Published online March 31, 2020. doi:10.1161/CIRCULATIONAHA.120.047063

11. Al-Khatib SM, Stevenson WG, Ackerman MJ, et al. 2017 AHA/ACC/HRS guideline for management of patients with ventricular arrhythmias and the prevention of sudden cardiac death: executive summary: a report of the American College of Cardiology/American Heart Association Task Force on Clinical Practice Guidelines and the Heart Rhythm Society.J Am Coll Cardiol . 2018;72:1677-1679.

12. Priori SG, Blomström-Lundqvist C, Mazzanti A, et al. 2015 ESC Guidelines for the management of patients with ventricular arrhythmias and the prevention of sudden cardiac death. Eur Heart $J$. 2015;36:2793-2867.

Figure. Priority plan for invasive cardiac electrophysiology procedures. Class I: benefit $>>>$ risk. Class IIa: benefit $>>$ risk. Class IIb: benefit [?] risk.

\begin{tabular}{|l|l|l|}
\hline $\begin{array}{l}\text { Guideline or Consensus } \\
\text { Statement Classification of } \\
\text { Recommendation }\end{array}$ & $\begin{array}{l}\text { Anticipated Short-term } \\
\text { Morbidity or Mortality } \\
\text { Benefit }\end{array}$ & Priority Category \\
\hline \multirow{4}{*}{ CLASS I } & High & I. Emergent/Urgent \\
\cline { 2 - 3 } & Intermediate & II. Equivocal \\
\cline { 2 - 3 } & Low or None & III. Elective \\
\hline \multirow{4}{*}{ CLASS IIa } & High & I. Emergent/Urgent \\
\cline { 2 - 3 } & Intermediate & II. Equivocal \\
\cline { 2 - 3 } & Low or None & III. Elective \\
\hline \multirow{3}{*}{ CLASS IIb } & Intermediate & II. Equivocal \\
\cline { 2 - 3 } & Low or None & III. Elective \\
\hline
\end{tabular}

